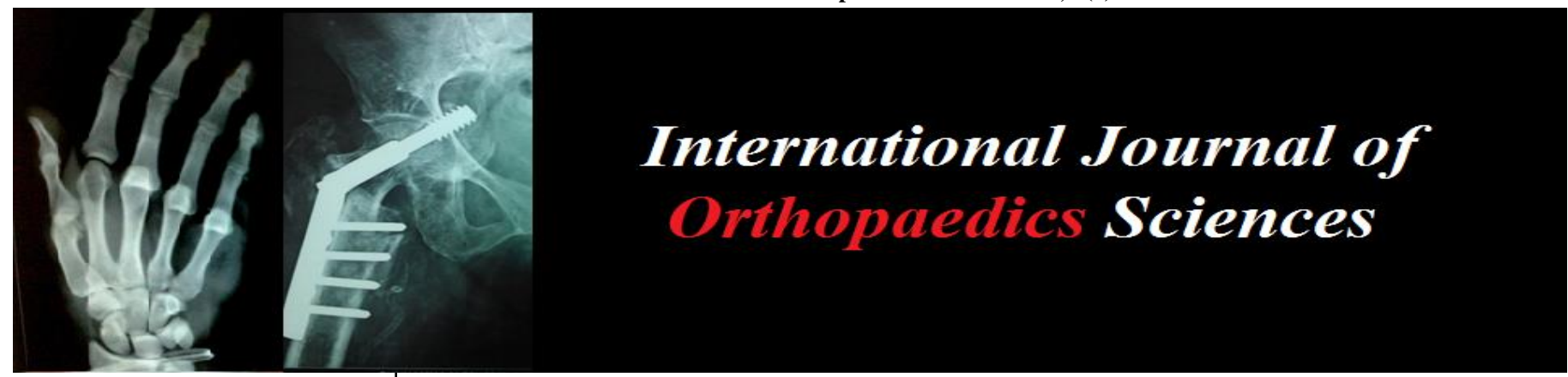

E-ISSN: 2395-1958

P-ISSN: 2706-6630

IJOS 2021; 7(3): 399-401

(C) 2021 IJOS

www.orthopaper.com

Received: 22-05-2021

Accepted: 24-06-2021

Dr. Prince Solomon

Pondicherry Institute of Medical

Sciences, Pondicherry,

Tamil Nadu, India

Dr. Siva Swaminathan

Santhanam

C-1, Staff Quarters, Pondicherry

Institute of Medical Sciences,

Ganapathichettikulam, Kalapet,

Pondicherry, Tamil Nadu, India

Dr. Gopi Kumarasamy

Pondicherry Institute of Medical

Sciences, Pondicherry,

Tamil Nadu, India

Dr. Ponnilavan Krishnan

Pondicherry Institute of Medical

Sciences, Pondicherry,

Tamil Nadu, India

Dr. Antony Albert

Pondicherry Institute of Medical

Sciences, Pondicherry,

Tamil Nadu, India

Dr. Brinda Ramanujam MOP Vaishnav College for Women, Chennai, Tamil Nadu, India
Corresponding Author: Dr. Siva Swaminathan Santhanam

C-1, Staff Quarters, Pondicherry Institute of Medical Sciences, Ganapathichettikulam, Kalapet, Pondicherry, Tamil Nadu, India

\section{Relationship between the timing of debridement of open fractures of lower extremity and the incidence of surgical site infection}

\author{
Dr. Prince Solomon, Dr. Siva Swaminathan Santhanam, Dr. Gopi \\ Kumarasamy, Dr. Ponnilavan Krishnan, Dr. Antony Albert and Dr. Brinda \\ Ramanujam
}

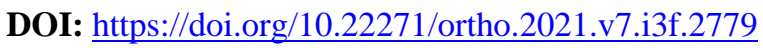

\begin{abstract}
The optimal timing of debridement of open fractures has been a debatable topic for many years. The purpose of the present study was to evaluate the relationship between the timing of initial debridement and the development of subsequent infection in open long bone fractures of lower limb presenting to our institution. We did a retrospective cohort study in 89 cases of open long bone fractures of lower limb who had underwent wound debridement and fracture fixation. Patients were grouped as Group A (44 patients) in which debridement of open fractures were done within 12 hours of injury and Group B (45 patients) wherein debridement was carried out after 12 hours of injury. Out of 44 patients in group A, 21 patients had infection and 23 had no signs of infection at the end of 1 year. In group B, out of 45 patients, 19 were infected and 26 had no signs of infection at 1year follow-up. Statistical analysis showed that there was no significant relationship between the timing of debridement and infection rate ( $p$ value -0.6 ). Hence open fractures can safely be debrided even after 12 hours of injury without increasing the chances of infection provided the antibiotics are started on time and the surgery is carried out by adequately rested and trained surgical team.
\end{abstract}

Keywords: open fractures, timing of debridement, infection

\section{Introduction}

The optimal timing of debridement of open fractures has not been clearly defined in the literature. In a country like India, due to logistical challenges, it is not possible to debride all open fracture within 6 to 12 hours of trauma. The concept of debridement of all open fractures within 6 hours of injury was established before the era of modern medicine, use of antibiotics and modern surgical techniques. American College of Surgeons Trauma Quality Improvement Program in 2015 recommended debridement of open fractures within 24 hours of injury ${ }^{[1]}$. Although emergency debridement is appropriate for open fractures, a time window for optimum treatment cannot be justified on the basis of existing literature ${ }^{[2]}$. Early debridement is often not feasible because of the need to transfer patients with complex injuries and because of ongoing resuscitative requirements in patients with multiple injuries. Debridement in the middle of the night with insufficient faculty may sometimes result in inadequate debridement too ${ }^{[3]}$. The purpose of the present study was to evaluate the relationship between the timing of initial debridement and the development of subsequent infection in open long bone fractures of lower limb presenting to our institution.

\section{Materials and Methods}

This was a retrospective cohort study with 102 patients. 5 patients died and 8 were lost during follow-up. 89 patients were included in this study at the final follow up. All patients with open long bone fractures of lower limb who had presented emergency department, undergoing wound debridement and fracture fixation were included in the study. Poly-trauma patients, patients younger than 16 years, open Gustilo and Anderson grade 1 fractures (only grade 2 and 3 included), incomplete case records and follow- up less than 1 year were excluded. 
Our standard treatment protocol for open fracture management included intravenous antibiotics at the time of presentation, early wound debridement and skeletal fixation and continuation of antibiotics 48 to 72 hrs after surgery. If a patient needed secondary plastic procedure or a conversion to definite orthopaedic fixation, it was done as and when needed. At the end of one year from the time of injury, the patients were assessed regarding the presence or absence of infection at the surgical site. A discharging sinus or a positive wound culture at the end of one year were taken as positive for infection. Patients were grouped as Group A (44 patients) in which debridement of open fractures were done within 12 hours of injury and Group B (45 patients) wherein debridement was carried out more than 12 hours after injury.

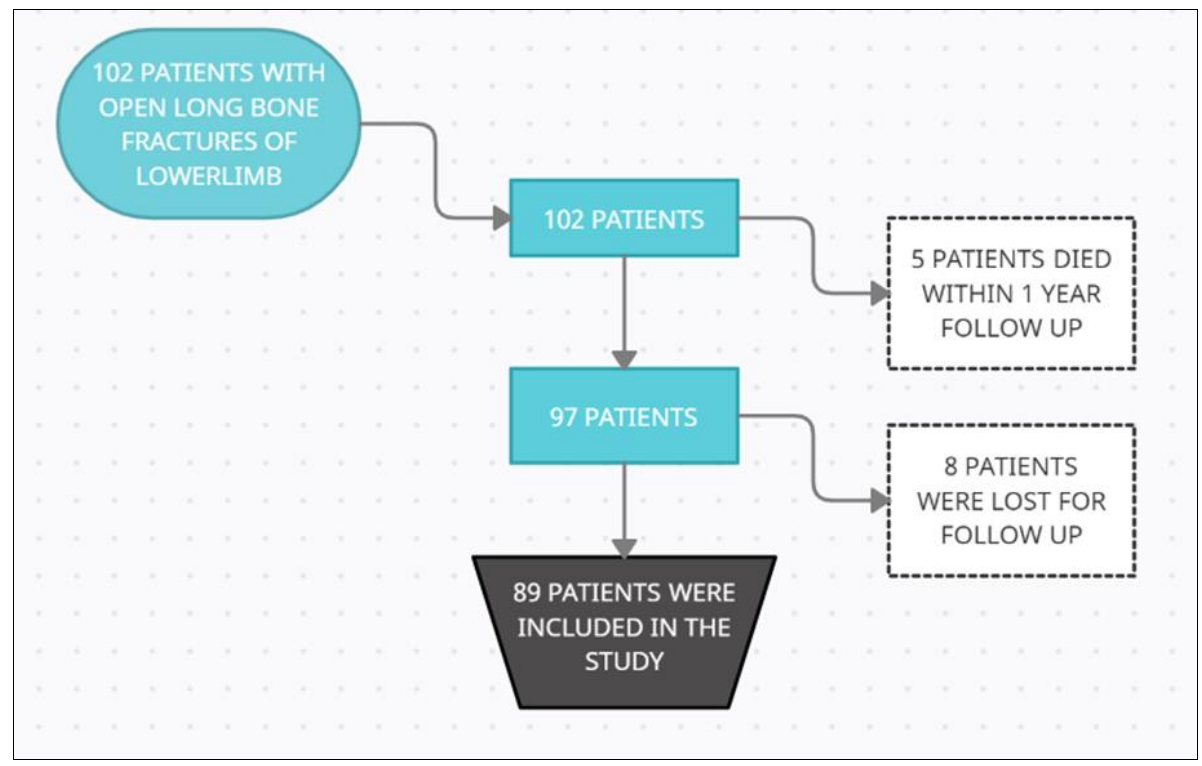

Fig 1: Patient flowchart

\section{Results}

89 patients were included in this study. 72 were male and the rest 17 were female patients. None of the female patients had a history of smoking whereas 38 male patients were smokers. Of the 38 smokers, only 6 had signs of infection at the end of one year and the correlation between smoking and infection was not found to be statistically significant. Out of 44 patients in group A, 21 patients had infection and 23 were normal at the end of 1 year. In group B, out of 45 patients, 19 were infected and 26 were normal at 1year follow-up. Statistical analysis showed that there was no significant relationship between the timing of debridement and infection rate ( $p$ value $-0.6)$.

\subsection{Statistical Analysis}

Table 1: Distribution of patients among the two groups

\begin{tabular}{|c|c|c|}
\hline Total $(\mathbf{n}=\mathbf{8 9})$ & Infected & Not infected \\
\hline Group A (Debrided $<12$ hours) $(\mathrm{n}=44)$ & 21 & 23 \\
\hline Group A (Debrided $>12$ hours) $(\mathrm{n}=45)$ & 19 & 26 \\
\hline
\end{tabular}

The chi square statistic is 0.27 . The p-value is 0.602 (not significant). The chi square statistic with Yates correction is 0.095 . The p-value is 0.76 (not significant).

\section{Discussion}

Although the treatment of open fractures should be on an urgent basis, in a developing country like India, wherein a tertiary care center with both an Orthopaedic and plastic team is not present in all district and villages, delay in treatment is inevitable. So, not all patients with open fractures can undergo an urgent debridement and skeletal fixation within 6 to 12 hours of injury. The age-old golden rule of debridement and skeletal stabilization of open fractures within 6 hours of injury is based on the theory published by Paul Leopold Friedrich ${ }^{[4]}$ in 1898 . He used garden mold and dust on guinea pigs and showed that the bacterial growth in maximum in the first 6 to 8 hours after contamination of a wound. So, it was recommended by him, that debridement should be carried out within 6 hours of injury. Kindsfater and Jonassen ${ }^{[5]}$ were able to show a significant increase in the rate of infection after a delay of six hours. Others, however, have been unable to support this period of time or any other time delay ${ }^{[5]}$. Kindsfater et al. studied 47 patients with grade 2 or grade 3 open tibial fractures and concluded that delaying debridement for more than 5 hours increased the chances of infection.

Several studies have been unable to show any difference between early and delayed debridement using infection as an end-point; Schenker et al. ${ }^{[6]}$ identified 3539 open fractures from 16 eligible studies. They concluded that there was no evidence to support a six-hour rule or any other particular threshold cut-off time to debridement. However, they also concluded that electing to delay the treatment of open fractures was not recommended. In our study, we retrospectively evaluated 89 patients with open grade 2 or grade 3 fracture of lower extremity (femur or tibia) by grouping them into group A (44 patients) wherein patients were debrided within 12 hours of injury and group B (45 patients) wherein patients underwent debridement after 12 hours of injury. Out of 44 in group A only 19 (43\%) had infection at the end of 1 year and out of 45 in group B, only 23 patients $(51 \%)$ had signs of infection. Statistical analysis showed a p value of 0.6 (Yates corrected - 0.76) implying no significant relationship between the timing of debridement and infection.

Khatod and colleagues ${ }^{[7]}$ found no increase in the infection rate for patients who received debridement in less than 6 hours vs those who were treated after 6 hours. Similarly, Tripuraneni and colleagues ${ }^{[8]}$ showed in a retrospective review of 206 patients with open tibia fractures that there was no difference in infectious outcomes based on debridement at less than 6 hours, 6 to 12 hours, and 12 to 24 hours. 
Enninghorst [9] and colleagues prospectively showed in a study of 89 patients that debridement of open tibia fractures within a mean of 8 hours, left injury severity as the only determinant of infectious complications among patients with blunt trauma. There was no difference in infectious complications between the early ( $<6$ hours) and late $(>6$ hours) debridement groups. In a retrospective study of 67 patients with grade III open tibia fractures, Singh et al. ${ }^{[10]}$ demonstrated no significant difference in the infection rate for early ( $<6$ hours) vs late ( $>6$ hours) debridement. Pollak ${ }^{[2]}$ showed in a prospective study of 307 patients with Gustilo grade III lower extremity open fractures that there was no difference in infectious complications among the groups. Merritt ${ }^{[11]}$ reported no significant relation between time to operative debridement and infection risk but a significant increase in infection related to longer than 121 minutes spent in the operating room.

Ours being a tertiary care centre in a second-tier city in India, usually has good amount of open fracture cases being referred from various neighbouring villages and districts. Most cases present late in the evening due to logistical issues. So, whether to take the case for debridement in the middle of the night or the next morning has always been a dilemma and our present study shows that debridement done even after 12 hours of injury does not increase the risk of infection. Taking a patient for debridement in the middle of the night by an already tired surgical team and inadequate theatre staff can lead to improper first debridement and can only worsen the situation.

We start the patient on triple antibiotics as soon as the patient presents to the emergency. As per the 2015 American College of Surgeons Trauma Quality Improvement Program recommendations on open fracture management, intravenous (IV) antibiotics should be started within 60 minutes of presentation to hospital and should be continued for not longer than 24 hours after surgical treatment, with the exception of severely contaminated fractures, where antibiotics can be continued upto 72 hours ${ }^{[1]}$.

\section{Conclusion}

This retrospective study shows that open fractures can safely be debrided even after 12 hours of injury without increasing the chances of infection provided the antibiotics are started on time and the surgery is carried out by adequately rested and trained surgical team. The golden rule of debridement within 6 hours of injury might not hold well in this modern era of orthopaedics.

\section{References}

1. You DZ, Schneider PS. Surgical timing for open fractures. OTA Int 2020;3(1):e067.

2. Pollak AN, Jones AL, Castillo RC, Bosse MJ, MacKenzie EJ. The Relationship between Time to Surgical Débridement and Incidence of Infection after Open High-Energy Lower Extremity Trauma. J Bone Joint Surg Am 2010;92(1):7-15.

3. Diwan A, Eberlin KR, Smith RM. The principles and practice of open fracture care 2018. Chin J Traumatol 2018;21(4):187-92.

4. Werner CML, Pierpont Y, Pollak AN. The Urgency of Surgical Débridement in the Management of Open Fractures. JAAOS - J Am Acad Orthop Surg 2008;16(7):369-75.

5. Hull PD, Johnson SC, Stephen DJG, Kreder HJ, Jenkinson RJ. Delayed debridement of severe open fractures is associated with a higher rate of deep infection. Bone Jt J 2014;96-B(3):379-84.

6. Schenker ML, Yannascoli S, Baldwin KD, Ahn J, Mehta S. Does timing to operative debridement affect infectious complications in open long-bone fractures? A systematic review. J Bone Joint Surg Am 2012;94(12):1057-64.

7. Khatod M, Botte MJ, Hoyt DB, Meyer RS, Smith JM, Akeson WH. Outcomes in Open Tibia Fractures: Relationship between Delay in Treatment and Infection: J Trauma Inj Infect Crit Care 2003;55(5):949-54.

8. The Effect of Time Delay to Surgical Debridement of Open Tibia Shaft Fractures on Infection Rate. Orthopedics 2008;31(12):01477447-20081201-27.

9. Enninghorst N, McDougall D, Hunt JJ, Balogh ZJ. Open Tibia Fractures: Timely Debridement Leaves Injury Severity as the Only Determinant of Poor Outcome. J Trauma Inj Infect Crit Care 2011;70(2):352-7.

10. Singh J, Rambani R, Hashim Z, Raman R, Sharma HK. The relationship between time to surgical debridement and incidence of infection in grade III open fractures. Strateg Trauma Limb Reconstr 2012;7(1):33-7.

11. Merritt K. Factors increasing the risk of infection in patients with open fractures. J Trauma 1988;28(6):823-7. 